\title{
Murray Valley encephalitis
}

\section{What is Murray Valley encephalitis?}

Murray Valley encephalitis (MVE) is a viral infection caused by the Murray Valley encephalitis virus. The disease is sometimes also called Australian encephalitis. Most people with this infection remain completely well and never develop any symptoms. Some people only develop a mild illness with fever. A small proportion of those infected develop a severe viral brain infection (encephalitis).

Some people with encephalitis develop permanent neurological complications. These complications can be fatal, especially when children are affected. About $40 \%$ of affected people make a complete recovery.

MVE is transmitted by infected mosquitoes and the disease usually occurs in remote north-western Australia. MVE has rarely been seen in eastern Australia.

\section{What are the symptoms?}

Only about 1 in 1000 people who are infected develop symptoms. Symptoms may include:

- fever

- headache

- rash

- myalgia (sore muscles)

- neck stiffness

- nausea, vomiting and diarrhoea

- seizures

- tremors

- lethargy, drowsiness, confusion and unconsciousness.

Symptoms appear 5-28 days after being bitten by an infected mosquito.

People with these symptoms should see a doctor promptly, especially if they live in, or have recently visited, an area that is known to have infected mosquitoes.

\section{How is it spread?}

The virus is spread by the common banded mosquito, Culex annulirostris. This mosquito breeds in fresh water and tends to be found in spring, summer and autumn around natural wetlands and irrigation waters. The mosquito is especially common around the Murray Darling River basin areas in NSW during summer.

This mosquito tends to be most active after sunset and around dawn. Some water bird species are also thought to be infected with the virus. Mosquitoes become infected by feeding on infected birds and possibly other animals. An infected mosquito can then bite a human and transmit the infection.

\section{Who is at risk?}

People at greatest risk include:

- People who have recently been bitten by mosquitoes.

- Babies, young children and newcomers to areas where the virus is active because they are less likely to have immunity from previous infection.

- Babies and young children are also more likely than adults to develop severe complications and to die if they become infected.

A person who is infected with the virus is not thought to be able to transmit the virus to another person.

People with previous infection are likely to be immune from re-infection, even if they were never sick.

\section{How is it prevented?}

The only protection from MVE is to avoid being bitten by mosquitoes. This is particularly important for travellers and visitors to areas where MVE might be active.

Protection from mosquitoes is essential:

- Avoid being outside when mosquitoes are most active, particularly around sunset, early evening and dawn.

- Wear loose-fitting, light-coloured clothing with long sleeves, long trousers and socks. Mosquitoes can bite through tight-fitting clothes.

- Use insect repellent when outdoors and reapply as directed by the manufacturer. Lotions and gels are more effective and longer lasting than sprays.

- Ensure flyscreens and doors are in good order.

- If camping, sleep under a mosquito net or in a mosquito-proof tent.

- Use a knock-down insect spray before going to bed to kill any mosquitoes that are indoors.

- Remember to protect babies and young children from mosquito bites too.

\section{How is it diagnosed?}

A blood test that detects antibodies to the virus can show if someone has had a recent or past infection. People with encephalitis often undergo a lumbar puncture (spinal tap) where the virus is detected in cerebro-spinal fluid.

\section{How is it treated?}

There is no specific treatment for MVE. People with encephalitis often require treatment in an intensive care unit. 
What is the public health response?

Laboratories are required to notify cases of MVE to the local public health unit. Public health unit staff interview the cases to identify risk factors. A single report will usually lead to a search for other possible cases and further advice to the local community.

NSW Health also performs surveillance for the virus in several locations in rural NSW by monitoring flocks of chickens for recent infection and by analysing trapped mosquitoes in summer months.
For more information please contact your doctor, local public health unit or community health centre.

This factsheet is available at: http://www.health.nsw.gov.au/ factsheets/infectious/influenza.html

\section{NSWOHEALTH}

